\title{
Mitochondrial remodeling in adipose tissue associated with obesity and treatment with rosiglitazone
}

Leanne Wilson-Fritch,1,2 Sarah Nicoloro, ${ }^{1,3}$ My Chouinard,,1,3 Mitchell A. Lazar, ${ }^{4}$ Patricia C. Chui, ${ }^{4}$ John Leszyk, ${ }^{3}$ Juerg Straubhaar, ${ }^{1}$ Michael P. Czech, ${ }^{1,3}$ and Silvia Corvera ${ }^{1,2}$

${ }^{1}$ Program in Molecular Medicine, ${ }^{2}$ Interdisciplinary Graduate Program, and ${ }^{3}$ Department of Biochemistry and Molecular Pharmacology, University of Massachusetts Medical School, Worcester, Massachusetts, USA. ${ }^{4}$ Division of Endocrinology, Diabetes and Metabolism, University of Pennsylvania, Philadelphia, Pennsylvania, USA.

\begin{abstract}
Adipose tissue plays a central role in the control of energy homeostasis through the storage and turnover of triglycerides and through the secretion of factors that affect satiety and fuel utilization. Agents that enhance insulin sensitivity, such as rosiglitazone, appear to exert their therapeutic effect through adipose tissue, but the precise mechanisms of their actions are unclear. Rosiglitazone changes the morphological features and protein profiles of mitochondria in 3T3-L1 adipocytes. To examine the relevance of these effects in vivo, we studied white adipocytes from $o b / o b$ mice during the development of obesity and after treatment with rosiglitazone. The levels of approximately $50 \%$ of gene transcripts encoding mitochondrial proteins were decreased with the onset of obesity. About half of those genes were upregulated after treatment with rosiglitazone, and this was accompanied by an increase in mitochondrial mass and changes in mitochondrial structure. Functionally, adipocytes from rosiglitazone-treated mice displayed markedly enhanced oxygen consumption and significantly increased palmitate oxidation. These data reveal mitochondrial remodeling and increased energy expenditure in white fat in response to rosiglitazone treatment in vivo and suggest that enhanced lipid utilization in this tissue may affect whole-body energy homeostasis and insulin sensitivity.
\end{abstract}

\section{Introduction}

The ability of brown adipose tissue to affect whole-body metabolism upon thermogenic stress has been known for many years $(1,2)$. More recently, the important contribution of white adipose tissue (WAT) to the control of energy homeostasis has been recognized with the study of tissue-specific knockout models and the discovery of adipocyte-specific secreted factors that have powerful effects on fuel metabolism $(3,4)$. These observations have raised many as-yetunanswered questions about the mechanisms by which adipocytes maintain their energy homeostasis as well as sense and respond to the metabolic requirements of the organism.

Brown adipocytes contain a large complement of mitochondria that serve as a source of heat generated as a consequence of flow through the electron transport chain under uncoupled conditions elicited by uncoupling protein 1 (UCP-1). Recently we reported that adipogenesis of the 3T3-L1 cell line, representative of white adipocytes, is also accompanied by a stimulation of mitochondrial biogenesis (5). The need for a large mitochondrial mass in white fat can be linked to key functions of the adipocyte that require mitochondrial function. For example, adipocytes must generate glycerol 3-phosphate at a rate sufficient to sustain triglyceride synthesis, and for this the glyceroneogenic pathway and

Nonstandard abbreviations used: CHAPS, 3-[(3-cholamidopropyl)dimethylammonio]-1-propanesulfonate; ESI, electrospray ion-trap; KRH, Krebs-Ringer solution buffered with HEPES; MALDI-TOF, matrix-assisted laser desorption ionization time-of-flight; UCP-1, uncoupling protein 1; UMMS-IACUC, University of Massachusetts Medical School Institutional Animal Care and Use Committee; WAT, white adipose tissue.

Conflict of interest: The authors have declared that no conflict of interest exists.

Citation for this article: J. Clin. Invest. 114:1281-1289 (2004).

doi:10.1172/JCI200421752. mitochondrial anaplerosis are critical $(6,7)$. In addition, the generation of acetyl-CoA for fatty acid activation prior to esterification into triglycerides may require a relatively large mitochondrial mass. Moreover, $\beta$-oxidation of fatty acids may be an important source of energy in the fat cell.

To further explore the role of white fat mitochondria, we have studied their abundance, composition, and morphology in cells derived from the epididymal fat pads of $o b / o b$ mice. In addition, we have analyzed the expression levels of mitochondrial genes in these cells. We found a profound decrease in the levels of transcripts for nuclear-encoded mitochondrial genes accompanying the onset of obesity. Strikingly, rosiglitazone treatment of $o b / o b$ mice reversed these effects and induced the expression of a set of genes normally silent in adipocytes from untreated animals. Direct analysis of the protein composition of mitochondria from rosiglitazone-treated animals mostly confirmed the information derived from RNA abundance measurements, but also revealed large changes in some proteins that were not reflected by their transcript levels. Functionally, these changes were apparent in the altered morphology of mitochondria as well as in the enhanced capacity of isolated cells to consume oxygen and oxidize palmitate. These studies provide new hypotheses to explain some of the therapeutic actions of rosiglitazone.

\section{Results}

Previous studies conducted in 3T3-L1 adipocytes revealed the induction of mitochondrial biogenesis during the process of differentiation, suggesting an important role for mitochondrial mass in white adipocyte function. Furthermore, exposure of fully differentiated 3T3-L1 adipocytes to rosiglitazone led to pronounced changes in mitochondrial morphology and to a small 

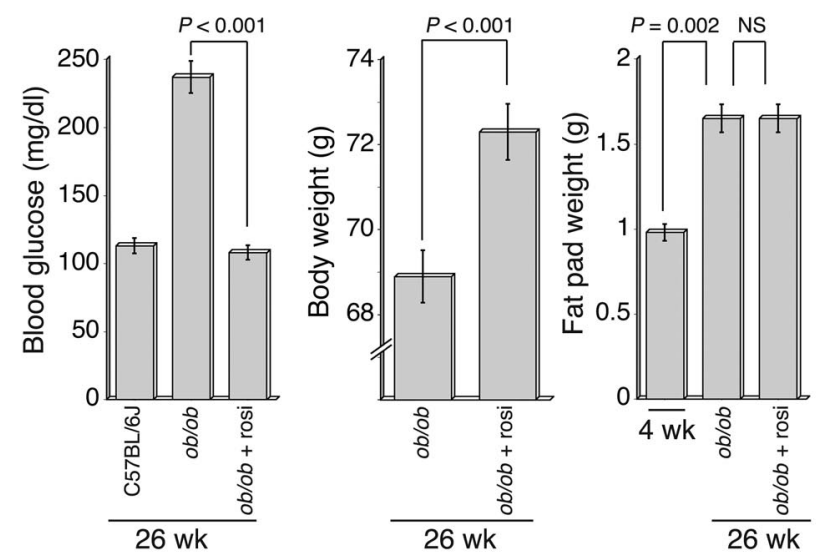

\section{Figure 1}

Effects of rosiglitazone on ob/ob mice. Fasting blood glucose concentrations, whole-body weight, and isolated fat pad weight were obtained in control animals or animals treated with rosiglitazone (Rosi) for 14 days. Data were derived from 10 independent experiments in which 2-5 mice of each condition were used.

but significant increase in mitochondrial mass. We searched for evidence for mitochondrial plasticity in vivo in white adipocytes isolated during the development of obesity and in response to rosiglitazone in $o b / o b$ mice.

Age-matched C57BL/6J and $o b / o b$ mice were used in this study. The effectiveness of rosiglitazone treatment was evidenced by the blood glucose concentration of $o b / o b$ mice, which decreased to that seen in age-matched C57BL/6J mice (Figure 1, left). During the 2 weeks of treatment of $o b / o b$ mice with rosiglitazone, a small but statistically significant increase in body weight was detected (Figure 1 , middle). Interestingly, while epididymal fat pad mass increased considerably between 4 and 26 weeks of age in $o b / o b$ animals, no statistically significant difference was detected in fat pad mass after rosiglitazone treatment (Figure 1, right).

Isolated adipocytes were obtained from epididymal fat pads from 26-week-old $o b / o b$ mice and age-matched C57BL/6J mice and were analyzed by fluorescence microscopy with an antibody against the mitochondrial chaperone $\mathrm{mHsp} 70$ (Figure 2). Cells obtained from 26-week-old $o b / o b$ mice were significantly larger than cells from age-matched C57BL/6J animals. In both cases, mitochondrial staining with anti-mHsp70 appeared as a punctate pattern distributed throughout the cytoplasm of the cell, with an increase in concentration near the perinuclear region, where the cytoplasmic volume is greatest. The average fluorescence intensities per cell from projected three-dimensional image stacks were $0.53,1.05$, and 1.58 units for C57BL6/J and $o b / o b$

\section{Figure 2}

Immunofluorescence analysis of mitochondrial Hsp70. Adipocytes were isolated from the epididymal fat pads of 26week-old C57BL/6J, ob/ob, and rosiglitazone-treated ob/ob $(o b / o b+$ Rosi) mice. Adipocytes were fixed and stained and image stacks were obtained with a conventional wide-field microscope fitted with a $40 \times$ or $100 \times$ Nikon plan-apo objective. Images represent single optical sections. White arrows indicate small, droplet-like structures seen only in rosiglitazone-treated cells. mice and for $a b / o b$ mice after rosiglitazone treatment. However, cells from $o b / o b$ mice had approximately twice the diameter of, and thus a surface area 4 times and volume 8 times larger than, cells from C57BL6/J mice. Assuming a requirement for mitochondrial mass proportional to cell size, these results indicate a deficit in mHsp70 staining in cells from $o b / o b$ mice relative to that of controls. Because average cell size did not differ between $o b / o b$ and rosiglitazone-treated $o b / o b$ mice, these results indicate an increase of approximately $50 \%$ in staining in cells from rosiglitazone-treated mice. The staining pattern of cells from rosiglitazone-treated animals differed markedly from that of cells from untreated $o b / o b$ mice in that it revealed a fine reticular pattern, also distributed throughout the cytoplasmic volume but significantly more concentrated in the perinuclear region (Figure 2). In addition, dense mitochondrial staining was observed surrounding small, droplet-like structures found in many cells from rosiglitazone-treated mice. The changes in mitochondrial density and morphology were also seen in cells stained with anti-mHsp60 (Figure 3); cells from rosiglitazone-treated animals contained almost twice the amount of mHsp60 per cell relative to cells from untreated animals. It is relevant that the staining pattern found in adipocytes from rosiglitazone-treated animals was seen in cells of a size equal to those from 26-week-old untreated animals, a finding that suggests that not only small cells (8) but also large cells within the adipose tissue are susceptible to the actions of rosiglitazone.

To rule out the possibility that the changes described above were due to rosiglitazone-induced alterations in mitochondrial chaperones, rather than to changes in true mitochondrial mass, we imaged live isolated adipocytes after incubating them with a specific indicator of mitochondrial mass, MitoTracker green. Mitochondria in age-matched C57BL/6J mice and 26-week-old ob/ob animals appeared as discrete punctate structures, while mitochondria in cells from $o b / o b$ mice treated with rosiglitazone were reticular and surrounded what appeared to be small lipid droplets present in many cells (Figure 4). Thus, rosiglitazone treatment in vivo results in pronounced changes in global mitochondrial mass and morphology. The presence of what appeared to be small lipid droplets surrounded by mitochondria suggests that considerable rearrangements of the lipid-metabolizing machinery of the white adipocyte occur in response to PPAR $\gamma$ stimulation by rosiglitazone.
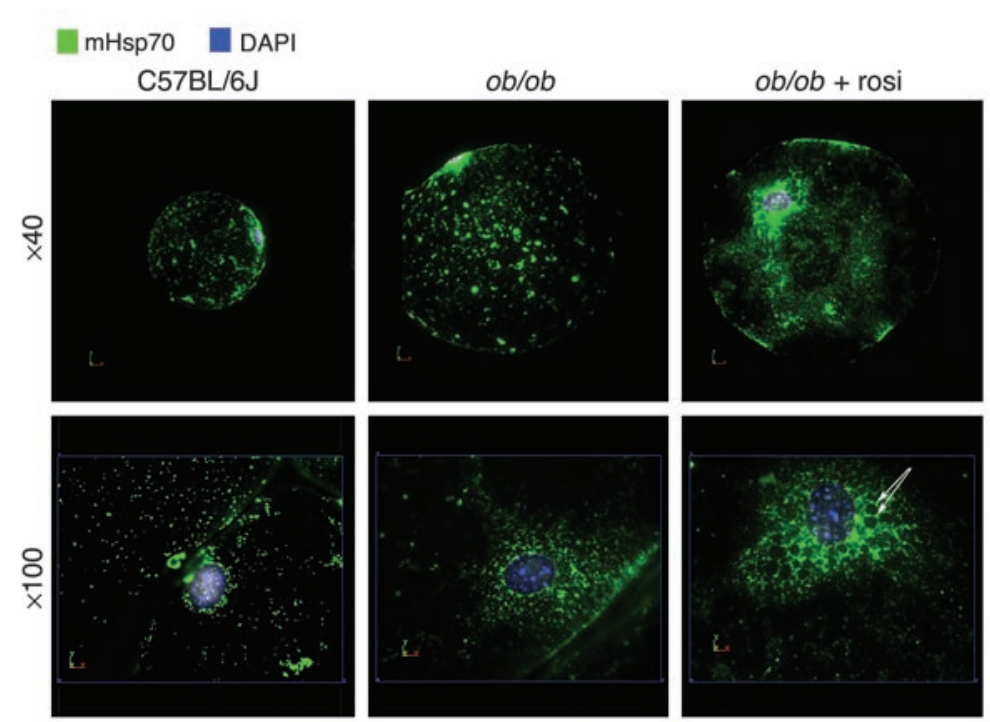

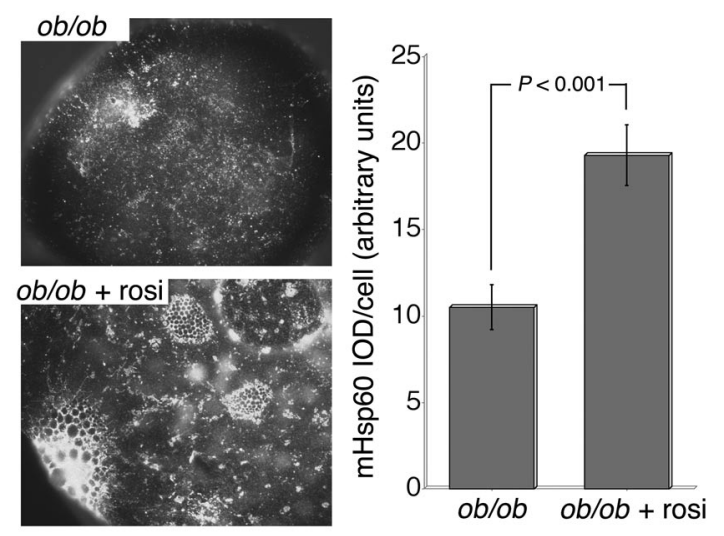

Figure 3

Immunofluorescence analysis of mitochondrial Hsp60. Isolated adipocytes from age-matched untreated and rosiglitazone-treated $o b / o b$ mice were fixed, stained, and imaged with a conventional widefield microscope fitted with a $40 \times$ or $100 \times$ Nikon plan-apo objective. Cell fluorescence intensity and statistical analyses were measured as described in Methods. IOD, integrated optical density.

To probe for the molecular basis of these morphological alterations, we surveyed a database of RNA expression levels obtained using Affymetrix chips (Figure 5). Of the approximately 36,000 genes and expressed sequence tag clusters in the murine genome $\mathrm{U} 74 \mathrm{v} 2$ probe set, 11,949 were detected as present in cells from 4and 26-week-old $o b / o b$ mice. The level of expression of 4,560 (38\%) of these genes differed between samples from 4- and 26-week-old mice. Of these, 2,245 (about 19\%) were increased in transcript level and 2,315 (about 19\%) were decreased. The database was then "queried" using a list of 700 probe sets previously annotated as "mitochondrial" in the Affymetrix database. Of these probe sets, 373 detected genes present in cells from 4- and 26-week-old $o b / o b$ mice. Approximately 204 (55\%) of these were significantly altered by the onset of obesity, with 23 (6\%) of genes found to be increased and 181 (49\%) found to be decreased. These results suggested that genes related to mitochondrial structure and function are overrepresented in the group of genes that decrease in expression level during the onset of obesity.

To determine how specifically this overrepresentation relates to genes of the mitochondria compared with those of other organelles, we used two additional lists of probe sets to "query" the database. One set consisted of 513 probe sets annotated with the term "endoplasmic reticulum" and another set consisted of 802 probe sets annotated with the term "nuclear." The "endoplasmic reticulum" probe set

\section{Figure 4}

Live cell imaging of mitochondria with MitoTracker Green FM. Adipocytes were isolated from epididymal fat pads of 26-week-old C57BL/6J or ob/ob mice that were either left untreated or were treated with rosiglitazone for 14 days. Cells were incubated with $100 \mathrm{nM}$ MitoTracker Green FM for 30 minutes and $25 \mu \mathrm{l}$ of the cell suspension was placed on a microscope slide. A glass coverslip was floated on the cell suspension and images were acquired within 5 minutes using a conventional wide-field microscope fitted with $a \times 40$ or $\times 100$ Nikon plan-apo objective. detected 249 genes present in cells from 4- and 26-week-old $o b /$ $o b$ mice. Of these, 99 (40\%) differed between samples from 4- and 26- week-old mice, with 47 (19\%) increased and 52 (21\%) decreased. The "nuclear" probe set detected 353 genes, of which 123 (35\%) differed between samples from 4- and 26-week-old mice, with 57 (16\%) increased and 66 (19\%) decreased. This similar frequency of genes displaying increased or decreased expression levels in the "endoplasmic reticulum" and "nuclear" probe set is similar to that seen for the "all-gene" probe set, but different from that seen for the "mitochondrial" set, for which genes displaying decreases in expression levels were 7-8 times more frequent than those displaying increases in expression levels.

The changes occurring after treatment with rosiglitazone in 26week-old mice were also analyzed (Figure 5). In the U75 probe set, 12,808 genes were detected as being present in cells from 26-weekold $o b / o b$ mice. The levels of expression of 1,539 (12\%) of these were altered by exposure to rosiglitazone. The expression levels of 1,102 (9\%) were increased and 437 (3.4\%) were decreased. The database was then "queried" with the same organelle-specific probe set lists described above. Of the probe sets annotated as "mitochondrial," 403 detected genes expressed in cells from 26-week-old ob/ob animals. Of these, 131 (32\%) were altered by rosiglitazone treatment, with 130 (32\%) displaying increased expression levels and only 1 $(0.2 \%)$ displaying a decreased expression level. Thus, genes associated with mitochondria are disproportionately affected by rosiglitazone treatment, with a strong bias toward an increase in gene expression. In contrast, other organelle-related genes displayed changes similar to those displayed by the total gene pool. Of the "endoplasmic reticulum" genes, 255 were detected in cells from 26 week-old animals. Of these, 28 (11\%) were altered by rosiglitazone treatment, with 18 (7\%) displaying increased expression and 10 (4\%) displaying decreased expression. Similarly, 391 "nuclear" genes were detected in cells from 26-week-old animals. Of these, 53 (14\%) were altered by rosiglitazone treatment, with 41 (11\%) displaying increased expression and 12 (3\%) displaying decreased expression.

In addition to causing a disproportionate increase in the expression of existing mRNAs for mitochondrial genes, rosiglitazone induced the expression of approximately 100 genes that were not detected in white adipocytes from 26-week-old $o b / o b$ mice. Among these are mitochondrial genes that would be expected to strongly influence energy metabolism and fatty acid oxidation and that are typically present in brown but not white adipocytes (Table 1). These include UCP-1, Cidea (9), and the M isoform of carnitine palmitoyltransferase I $(10,11)$.
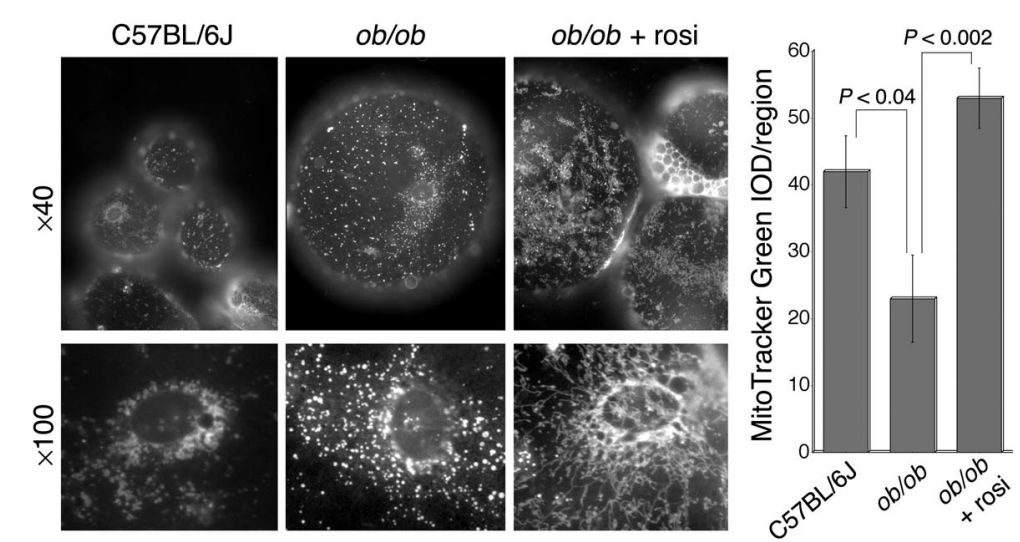


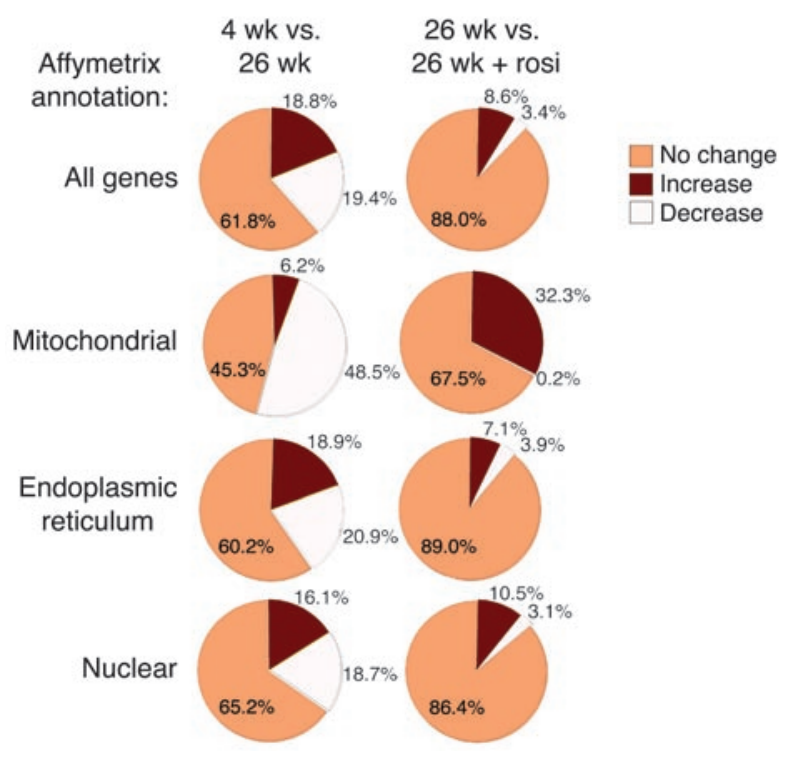

To survey the degree of concordance between the changes in mRNA levels and changes at the protein level, we purified mitochondria from isolated adipocytes, solubilized them, and analyzed them by sucrose gradient centrifugation and SDS-PAGE. When normalized to the initial protein content of the cell homogenates from which mitochondria were isolated, the yield of mitochondrial protein was approximately double from cells from rosiglitazonetreated $o b / o b$ mice compared with untreated age-matched controls. This increase yield is consistent with the doubling of mitochondrial mass on a per-cell basis deduced by mitochondrial staining (Figure 2 ). The more abundant proteins that displayed changes in response to rosiglitazone were excised and identified by mass spectrometry (Figure 6). We then "queried" the Affymetrix dataset to determine the expression pattern of the mRNAs for these specific proteins (Table 2). As expected, concordant increases in protein and mRNA levels in response to rosiglitazone were observed for many of the proteins identified by mass spectrometry. However, the increases in protein abundance exceeded the increased level of expression detected by the Affymetrix chip analysis in several instances. For example, a 2- to 3-fold increase in protein levels of malate dehydrogenase (Mor1) corresponded to a 1.45 -fold increase in mRNA abundance. More strikingly, the mitochondrial chaperones $\mathrm{mHsp} 70$ and mHsp60 were not "scored" as present in mouse adipose cells in the Affymetrix chip despite being abundant mitochondrial proteins, as judged by mass spectrometry. Moreover, the rosiglitazone-induced increase in the level of chaperone expression, which was evident both by mass spectrometry (Figure 6) and Western blotting (Figure 7), was not detected by the Affymetrix chip analysis. This discordance suggests that mitochondrial protein expression may be particularly subject to regulation at the post-translational level and that some of the actions of rosiglitazone on mitochondrial structure/function may take place through translational control.

Among the proteins increased in response to rosiglitazone are several key proteins of the fatty acid $\beta$-oxidation pathway. Notably, the protein levels of short-chain, medium-chain, long-chain, and very-long-chain acyl-CoA dehydrogenases, which catalyze the key reaction in the $\beta$-oxidation of fatty

\section{Figure 5}

Analysis of gene expression changes in adipocytes from ob/ob mice. Total RNA was extracted from isolated adipocytes from 4-week-old (4 week) untreated, 26-week-old (26 week) untreated, and 26-week-old rosiglitazone-treated ob/ob mice and was used to probe the murine U74v2 Affymetrix GeneChip. The resulting data were queried using probe annotations within the Affymetrix database. vs., versus.

acids, were found to be increased by rosiglitazone. Reducing equivalents from these dehydrogenases are transported to the main respiratory chain by the electron transfer flavoprotein, composed of two subunits, Etfa and Etfb, and by the electron transfer flavoprotein dehydrogenase $E t f d h$ (12). The levels of all three of these proteins were detected to be increased by mass spectrometry and Affymetrix chip analysis. These results suggest that $\beta$-oxidation of fatty acids might be increased in response to rosiglitazone. To directly test this hypothesis, we measured the conversion of $\left[{ }^{14} \mathrm{C}\right]$ palmitate to $\left[{ }^{14} \mathrm{C}\right] \mathrm{O}_{2}$ by isolated adipocytes derived from control and $o b / o b$ mice. A significant increase in palmitate oxidation was detected in cells from rosiglitazone-treated animals (Figure 8).

Additional evidence for the functional relevance of the observed alterations in mitochondrial mass and composition in obesity is the marked depression in oxygen consumption by isolated adipocytes from $o b / o b$ mice compared with that of cells from 4-week-old $o b / o b$ animals (Figure 9) or age-matched C57BL/6J mice (data not shown). This depressed oxygen consumption rate was almost completely reversed in cells from rosiglitazone-treated mice (Figure 9).

To investigate the potential mechanisms involved in the observed changes in mitochondrial mass and function in response to rosiglitazone, we analyzed the levels of PGC- $1 \alpha$ (Figure 10). While the transcript levels for this coactivator were below the limits of detection in the Affymetrix chip, direct Northern

\section{Table 1}

Rosiglitazone-induced mitochondrial gene expression in ob/ob mouse adipocytes

\section{Gene name}

Uncoupling protein 1, mitochondrial Uncoupling protein 1, mitochondrial Uncoupling protein 1, mitochondrial Cytochrome c oxidase, subunit VIIIb Cytochrome c oxidase, subunit VIIIb Cytochrome $c$ oxidase, subunit VIla 1 Pyruvate carboxylase Pyruvate carboxylase Isocitrate dehydrogenase $3\left(N A D_{+}\right) \beta$ Isocitrate dehydrogenase $3(N A D+) \beta$

Electron transfer flavoprotein dehydrogenase

Cytochrome c, somatic

Carnitine palmitoyltransferase 1, muscle Carnitine palmitoyltransferase 1, muscle Uncoupling protein 1, mitochondrial

$\begin{array}{cccc}\text { Gene symbol } & \text { FC } & \text { Call } & \text { Change } \\ \text { Ucp1 } & 20.63 & \mathrm{P} & \mathrm{I} \\ \text { Ucp1 } & 21.61 & \mathrm{P} & \mathrm{I} \\ \text { Ucp1 } & 4.7 & \mathrm{P} & \mathrm{I} \\ \text { Ucp1 } & 4.7 & \mathrm{P} & \mathrm{I} \\ \text { Cox8b } & 4.29 & \mathrm{P} & \mathrm{I} \\ \text { Cox8b } & 3.25 & \mathrm{P} & \mathrm{I} \\ \text { Cox7a1 } & 5.53 & \mathrm{P} & \mathrm{I} \\ \text { Pcx } & 3.17 & \mathrm{P} & \mathrm{I} \\ \text { Pcx } & 2.58 & \mathrm{P} & \mathrm{I} \\ \text { Idh3b } & 2.52 & \mathrm{P} & \mathrm{I} \\ \text { Idh3b } & 2.46 & \mathrm{P} & \mathrm{I} \\ \text { Etfdh } & 2.35 & \mathrm{P} & \mathrm{I} \\ & & & \\ \text { Cycs } & 2.35 & \mathrm{P} & \mathrm{I} \\ \text { Cpt1b } & 4.92 & \mathrm{P} & \mathrm{I} \\ \text { Cpt1b } & 4.7 & \mathrm{P} & \mathrm{I}\end{array}$

The genes listed were expressed at levels low enough to qualify as absent from the cells from 4-week-old and 26-week-old ob/ob mice but present in those from 26-week-old rosiglitazone-treated mice. FC, "fold change" in gene expression; Call, presence $(P)$ or absence $(A)$ of probe on the GeneChip; Change, increase (I) or lack of change (NC) in expression in response to rosiglitazone. 
blotting revealed a signal for PGC-1 $\alpha$, which was increased significantly in response to rosiglitazone.

\section{Discussion}

The use of the thiazolidinediones rosiglitazone and pioglitazone for the treatment of type 2 diabetes is firmly established. Both drugs result in a net improvement in insulin sensitivity, and recent evidence suggests beneficial effects of these drugs on cardiovascular parameters associated with metabolic syndrome and type 2 diabetes complications $(13,14)$. The mechanism of action of rosiglitazone and pioglitazone is centered on their ability to activate PPAR $\gamma$, which is abundantly expressed in adipose tissue and is present in vasculature, colonic epithelium, and leukocytes. However, the relationship between PPAR $\gamma$ activation and the physiological and therapeutic actions of the glitazones is not completely clear. The work presented here suggests that rosiglitazone, presumably through its stimulatory effect on PPAR $\gamma$, induces mitochondrial biogenesis and remodeling in WAT in a way that enhances fatty acid oxidation and markedly enhances oxygen consumption. This effect of rosiglitazone can be envisioned to contribute directly and indirectly to changes in wholebody energy metabolism and insulin sensitivity.

The salient effects of rosiglitazone treatment were a doubling of total mitochondrial mass and the additionally increased levels of mitochondrial proteins expected to enhance glyceroneogenesis, fatty acid transport into the mitochondrial matrix, fatty acid oxidation, and electron transport. In addition, further enhanced flux through the electron transport chain would be expected to result from the greatly increased UCP-1 message levels. The consequences of these changes are evidenced functionally by the increase in palmitate oxidation and greatly enhanced rate of oxygen consumption by isolated adipocytes from rosiglitazone-treated animals. These results raise two important questions. First, does this effect of rosiglitazone on white fat mitochondrial mass and energy metabolism lead to the enhancement of whole-body insulin sensitivity that is the hallmark of the drug's therapeutic effect? Sec-

\section{Table 2}

The mRNA expression levels of proteins identified by mass spectrometry

\begin{tabular}{|c|c|c|c|c|c|c|}
\hline Accession $n$ & Gene name & Gene symbol & Affymetrix probe sets & FC & Call & Change \\
\hline 477004 & Epoxide hydrolase 2, cytoplasmic & Ephx2 & 93051_at & 4.39 & $P$ & I \\
\hline 200246 & Pyruvate carboxylase & $P C X$ & 93308_s_at & 1.29 & $P$ & I \\
\hline 200246 & Pyruvate carboxylase & $P C X$ & AFFX-PyruCarbMur/L09192_3_at & 1.29 & $\mathrm{P}$ & NC \\
\hline 200246 & Pyruvate carboxylase & Pcx & AFFX-PyruCarbMur/L09192_5_at & 3.17 & $\mathrm{P}$ & I \\
\hline 200246 & Pyruvate carboxylase & $P C X$ & AFFX-PyruCarbMur/L09192_MA_at & 2.58 & $\mathrm{P}$ & I \\
\hline 200246 & Pyruvate carboxylase & $P C X$ & AFFX-PyruCarbMur/L09192_MB_at & 2.05 & $P$ & I \\
\hline 200246 & Pyruvate carboxylase & $P C X$ & 162288_f_at & 1.05 & $\mathrm{P}$ & NC \\
\hline 200246 & Pyruvate carboxylase & $P C X$ & 168162_f_at & 1.35 & $P$ & NC \\
\hline 200246 & Pyruvate carboxylase & $P C X$ & 171076_i_at & -2.14 & A & NC \\
\hline 21313290 & Electron transferring flavoprotein, dehydrogenase & Etfdh & 97869_at & 2.35 & $\mathrm{P}$ & I \\
\hline 13385680 & 2,4,-Dienoyl-CoA reductase & Decr1 & 115824_at; 160711_at & 1.9 & $P$ & I \\
\hline 19684166 & Acyl-coenzyme A dehydrogenase, short chain & Acads & 103401_at & 1.87 & $\mathrm{P}$ & I \\
\hline 19684166 & Acyl-coenzyme A dehydrogenase, short chain & Acads & 161469_r_at & -1.15 & $P$ & NC \\
\hline 19684166 & Acyl-coenzyme A dehydrogenase, short chain & Acadsb & 162725_at & 1.12 & $P$ & NC \\
\hline 32130423 & Acetyl-coenzyme A dehydrogenase, long-chain & Acadl & 95425_at & 1.82 & $\mathrm{P}$ & I \\
\hline 21759113 & Electron transferring flavoprotein, alpha polypeptide & Etfa & 96112_at & 1.82 & $\mathrm{P}$ & I \\
\hline 5304852 & Aconitase 2, mitochondrial & Aco2 & 96870_at & 1.62 & $P$ & I \\
\hline 181575 & Dihydrolipoamide dehydrogenase & Dld & 97502_at & 1.48 & $P$ & I \\
\hline 42476181 & Malate dehydrogenase, mitochondrial & Mor1 & 93991_at & 1.45 & $P$ & । \\
\hline 21431780 & L-3-hydroxyacyl-CoA dehydrogenase, short chain & Hadhsc & 95485_at & 1.45 & $P$ & I \\
\hline 13386272 & Citrate synthase & Cs & 99666_at & 1.38 & $\mathrm{P}$ & 1 \\
\hline 13386272 & Citrate synthase & Cs & 164575_f_at & 1.87 & A & I \\
\hline 30583785 & Isocitrate dehydrogenase $3(N A D+)$ alpha & Idh3a & 94534_at & 1.35 & $P$ & I \\
\hline 30583785 & Isocitrate dehydrogenase $3(N A D+)$ alpha & Idh3a & 169744_i_at & -1.02 & A & NC \\
\hline 30583785 & Isocitrate dehydrogenase $3(N A D+)$ alpha & Idh3a & 170915_r_at & -1.07 & A & NC \\
\hline 29351581 & Electron transferring flavoprotein, beta polypeptide & Etfb & 162144_at & -1.38 & A & NC \\
\hline 29351581 & Electron transferring flavoprotein, beta polypeptide & Etfb & 96947_at & 1.35 & $\mathrm{P}$ & NC \\
\hline 6678970 & Methylmalonyl-coenzyme A mutase & Mut & 99613_at & 1.23 & $\mathrm{P}$ & I \\
\hline 23956084 & Acyl-coenzyme A dehydrogenase, very long chain & Acadvl & 161623_at & -1.91 & A & NC \\
\hline 23956084 & Acyl-coenzyme A dehydrogenase, very long chain & Acadvl & 93334_at & 1.18 & A & NC \\
\hline 6753036 & Aldehyde dehydrogenase 2, mitochondrial & Aldh2 & 96057_at & 1.07 & $\mathrm{P}$ & NC \\
\hline 6753036 & Aldehyde dehydrogenase 2 , mitochondrial & Aldh2 & 96058_s_at & 1.05 & $P$ & NC \\
\hline 729927 & Fatty acid coenzyme A ligase, long chain 2 & Facl2 & 94507_at & 1.05 & $\mathrm{P}$ & NC \\
\hline 14917005 & mHsp70 & Hspa9a & 97914_at & 2.19 & A & NC \\
\hline 14917005 & mHsp70 & Hspa9a & 170001_at & 1.26 & $P$ & I \\
\hline 14917005 & mHsp70 & Hspa9a & 171404_r_at & 1.15 & A & NC \\
\hline 17391295 & $m H s p 60$ & Hspd1 & 161140_r_at & 1.41 & $\mathrm{P}$ & NC \\
\hline 17391295 & $m H s p 60$ & Hspd1 & 93277_at & -1.2 & A & NC \\
\hline
\end{tabular}

The Affymetrix GeneChip database was queried for the genes encoding the proteins identified by mass spectrometry described in Figure 5. 


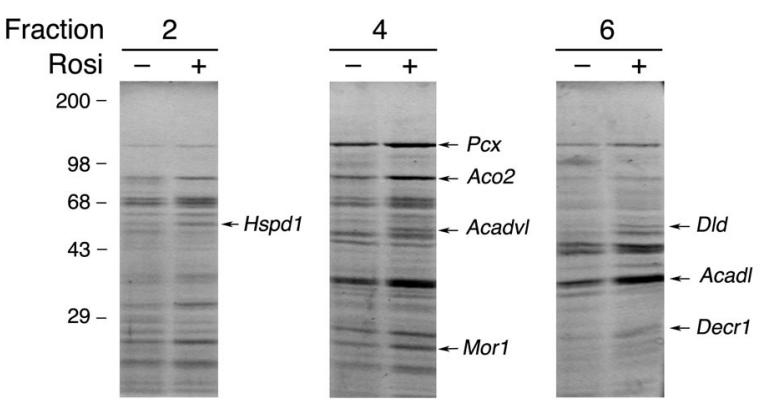

Figure 6

Comparative analysis of mitochondrial protein expression in untreated and rosiglitazone-treated adipocytes. Mitochondria isolated from adipocytes from untreated (-) and rosiglitazone-treated (+) ob/ob mice were analyzed by sucrose gradient centrifugation and SDS-PAGE. Proteins exhibiting substantial differential expression in three independent experiments were excised and identified by mass spectrometry. Illustrated is one experiment from which the indicated proteins were excised. They are identified along the left margins by their respective gene symbols.

ond, what is the molecular basis for the effect of rosiglitazone on mitochondrial mass and patterning?

Numerous lines of evidence support the notion that increased circulating free fatty acids (FFAs) directly cause insulin resistance. The levels of circulating FFAs are influenced not only by the rate of triglyceride lipolysis but also by the rate of re-esterification and oxidation within the fat cell (15). Indeed, it has been calculated that as much as $40 \%$ of FFAs are re-esterified after lipolysis in adipose tissue from lean adults (16). The re-esterification rate appears to be rate limited by the levels of enzymes of glyceroneogenesis, as it is greatly increased by tissue-specific overexpression of phosphoenolpyruvate carboxykinase (PEPCK). These precedents suggest that the glyceroneogenesis pathway may be rate limiting for FFA re-esterification and the enhancement of mitochondrial mass by rosiglitazone is likely to result in enhanced production of glyceroneogenic substrates and re-esterification. Indeed, an additional increase in the protein levels of pyruvate kinase within purified mitochondria was seen in this study (Figure 6), and re-esterification in response to rosiglitazone has been observed in adipocytes from rosiglitazone-treated $o b / o b$ animals (S. Corvera and M.P. Czech, unpublished observations).

In addition to enhancement of re-esterification, an important effect of rosiglitazone on fatty acid oxidation cannot be ruled out and is supported by the results obtained in this study. In our experiments, adipocytes from rosiglitazone-treated animals displayed an increase of about $30 \%$ in palmitate oxidation, a value that is likely to be an underestimate of the true effect of rosiglitazone in the face of enhanced triglyceride/fatty acid cycling. The fraction of FFAs that are oxidized within the fat cell has been measured to be only $0.2 \%$ of that produced by lipolysis. However, this increases to $0.5 \%$ during fasting and is highly suppressed by insulin (15). Moreover, the high capacity for white fat to oxidize fatty acids under hyperleptinemic conditions has been reported (17). Thus, FFA oxidation in WAT is regulated, and a thiazolidinedione-induced shift toward an increase oxidation over time could have a pronounced effect on circulating FFA levels and whole-body insulin sensitivity. Interestingly, a previously observed unusual finding is the accumulation of 22:6n3 and other long-chain polyunsaturated fatty acids in the triglyceride pool of adipose tissue in response to rosi- glitazone (18). This unusual response may be explained by the fact that mitochondria, as compared with the peroxisome, cannot oxidize polyunsaturated fatty acids. Thus, under conditions of rapid triglyceride/FFA cycling and enhanced mitochondrial $\beta$-oxidation, polyunsaturated fatty acids would accumulate.

An increase in mitochondrial content, respiration, and fatty acid oxidation in WAT would be expected to cause a decrease in adiposity. Paradoxically, animals treated with rosiglitazone displayed a very small but significant increase in body weight, consistent with the known effect of PPAR $\gamma$ activation to induce adipogenesis. The increase in body weight was not accompanied by a detectable increase in the weight of the epididymal fat pads, suggesting that it may be due to fat accumulation in other depots. Interestingly, a "redistribution" of fat from visceral to peripheral depots has been observed to occur in response to treatment with thiazolidinediones in rodents and humans (19-23). These results suggest the hypothesis that rosiglitazone produces two simultaneous effects: one to increase adipogenesis and second to increase fat utilization by increasing mitochondrial mass, fatty acid oxidation, and oxygen consumption. These two effects may be differentially elicited in a depot-specific manner, leading to an overall increase in body weight while decreasing visceral adiposity. Further work will be required to directly test this hypothesis. Interestingly, pioglitazone has been reported to enhance daily profiles of energy expenditure and lipid utilization in diabetic rats with no changes in body weight (24), suggesting differences between animal models in the predominant responses to thiazolidinediones.

A link between insulin resistance and the mitochondrial oxidative phosphorylation pathway has recently emerged in the analysis of genes expressed in human populations of insulin-resistant individuals $(25,26)$. Furthermore, insulin resistance in elderly patient populations is associated with striking decreases in mitochondrial oxidative phosphorylation (27). However, a causative link between decreased expression of genes that encode proteins involved in oxidative phosphorylation and the development of insulin resistance has not been demonstrated. The results shown here support the
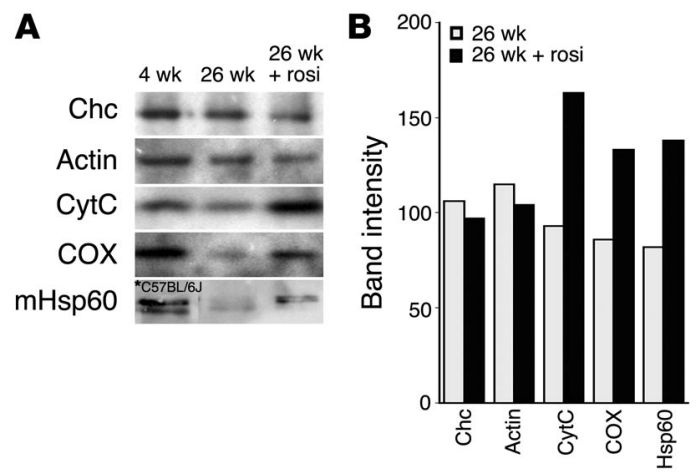

\section{Figure 7}

Western blotting of mitochondrial proteins. Isolated adipocytes were lysed in sample buffer containing $1 \%$ SDS. Equal concentrations of total protein $(20 \mu \mathrm{g})$ were analyzed by SDS-PAGE and Western blotting using antibodies against mitochondrial (CytC, cytochrome $c$; COX, cytochrome oxidase subunit II; $\mathrm{mHsp60}$ ) and non-mitochondrial (Chc, clathrin heavy chain; Actin) proteins. Except where indicated by the asterisk, all samples were from ob/ob mice with the ages and conditions indicated. Band intensities were quantified using Photoshop 7 software as described in Methods and were plotted. Similar results were obtained in three experiments. 


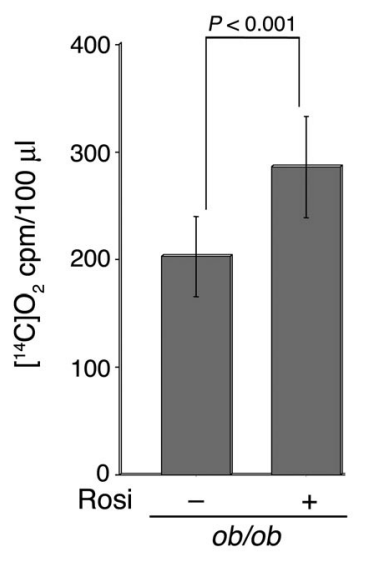

Figure 8

Fatty acid oxidation in isolated adipocytes. Adipocytes were isolated from 26-week-old untreated or rosiglitazone-treated ob/ob mice. Palmitate oxidation was estimated by the measurement of $\left[{ }^{14} \mathrm{C}\right] \mathrm{O}_{2}$ release over a 2-hour period as described in Methods. The numbers of cells present in $100 \mu \mathrm{l}$ of packed volume, estimated by direct DNA measurements, were $5.4 \times 10^{5}$ and $5.1 \times 10^{5}$ in the absence and presence of rosiglitazone treatment, respectively.

idea of such a causative role by demonstrating that a rosiglitazone, a prototypical member of a class of drugs that improve insulin sensitivity and elicit alterations in mitochondrial proteins and in the cDNAs for mitochondrial genes that are nuclear encoded, would be predicted to enhance flow through the electron transport chain and oxidative phosphorylation.

Important insights into the mechanisms involved in the establishment of cellular mitochondrial mass have been obtained in recent years (28). Salient among these is the realization of the role of the transcriptional coactivator PGC- 1 in the stimulation of the mitochondrial biogenesis program in brown fat and muscle cells (29). Despite the fact that endogenous PGC-1 levels are very low in WAT and that the interaction between PPAR $\gamma$ and PGC-1 has been thought to be ligand independent (30), it nevertheless is possible that rosiglitazone, by increasing the levels of PGC- $1 \alpha$ and through activation of PPAR $\gamma$, enhances coactivation of nuclear respiratory factors by the small amount of PGC-1 present, enough to induce expression of some mitochondrial genes, such as UCP-1. This in turn might lead to further expansion of mitochondrial mass through as-yet-unclear mechanisms (31). An increase in expression of brown fat-specific mitochondrial genes has been seen in response to exogenous insertion of PGC- 1 into human adipocytes (32), consistent with the possibility that activation of PGC-1 in this tissue can enhance mitochondrial biogenesis and remodeling. Alternatively, additional PPAR $\gamma$ ligand-dependent mechanisms for induction of nuclear-encoded mitochondrial genes may remain to be discovered.

\section{Methods}

Animals. All procedures were carried out following guidelines of the University of Massachusetts Medical School Institutional Animal Care and Use Committee (UMMS-IACUC). The 4-week-old male ob/ob, 10-week-old male $o b / o b$, and 10-week-old male C57BL/6J mice were purchased from Jackson Laboratory and were housed ( $n=4$ per cage) under 12-hour light/dark cycles with ad libitum access to food and water. The 10-week-old mice were allowed to age to 26 weeks on standard pellet mouse chow. Prior to 26 weeks of age, half of the animals were treated with $0.375 \mathrm{mg} /$ day of Avandia rosiglitazone maleate tablets (SmithKline Beecham Pharmaceuticals) for 14 days, delivered in powdered, standard lab mouse chow ad libitum. Rosiglitazone was ground in a coffee grinder and was added to the powdered mouse chow. Control animals not treated with rosiglitazone were given powdered standard lab chow ad libitum. Animals were made to fast overnight for 18 hours prior to tissue harvesting. Animals were sacrificed according to guidelines from UMMS-IACUC-approved protocols, and epididymal fat pads were harvested from the mice and placed in Krebs-Ringer solution buffered with HEPES (KRH), pH 7.4, supplemented with 2.5\% BSA.

Blood glucose monitoring. Fasting blood glucose concentrations were determined from whole venous blood obtained from the pleural cavity using the Accu-chek Advantage blood glucose monitoring system (Roche Diagnostics).

Primary fat cell isolation. Fat pads were weighed and cut into $0.3-\mathrm{cm}^{3}$ pieces with scissors and then were resuspended in $\mathrm{KRH}, \mathrm{pH} 7.4$, plus $2.5 \%$ BSA containing $1 \mathrm{mg} / \mathrm{ml}$ collagenase (Worthington Biochemical Corp.). The fat pads were digested for $30-45$ minutes at $37^{\circ} \mathrm{C}$ in an orbital bath shaking at $100 \mathrm{rpm}$. The digested samples were squeezed through chiffon material and the isolated cells were washed 3 times in KRH buffer, pH 7.4, plus 2.5\% BSA.

Fluorescence microscopy. Adipocytes were washed two times in PBS and were fixed for 1 hour at $4{ }^{\circ} \mathrm{C}$ in $4 \%$ formaldehyde in PBS. Cells were then permeabilized for 20 minutes at room temperature in PBS plus $0.5 \%$ Triton $\mathrm{X}-100$ and then were blocked for 30 minutes at $4^{\circ} \mathrm{C}$ in PBS plus $0.5 \%$ Triton X-100 plus $1 \%$ FBS. Cells were incubated overnight at $4^{\circ} \mathrm{C}$ with the indicated primary antibodies in fresh blocking solution. Cells were washed with blocking solution and were incubated for 30 minutes at room temperature with anti-mouse or anti-rabbit IgG HRP-conjugated secondary antibody (Promega). Cells were washed with PBS and were incubated for 15 minutes at room temperature with $2 \mu \mathrm{g} / \mathrm{ml}$ DAPI stain. Cells were washed with PBS and mounted on slides using the Prolong Antifade Kit (Molecular Probes) and were imaged with a conventional wide-field microscope fitted with a $60 \times$ or $100 \times$ Nikon PlanApo objective. Stacks of 50 optical sections

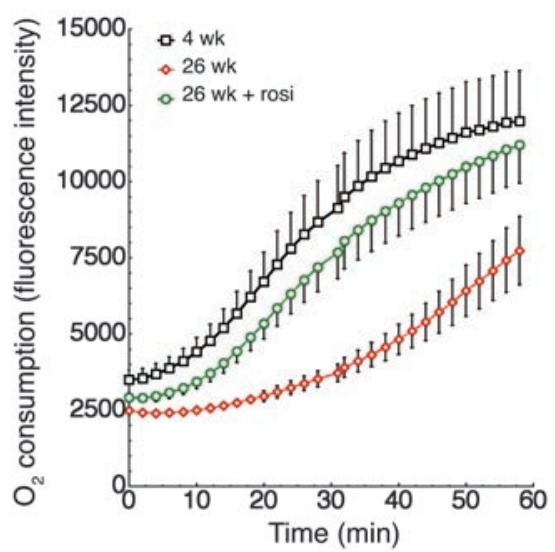

\section{Figure 9}

Oxygen consumption in isolated adipocytes. Adipocytes were isolated from 4-week-old, 26-week-old untreated, or 26-week-old rosiglitazonetreated ob/ob mice. Equal volumes of packed cells were separated into aliquots in wells of a 96-well BD Oxygen Biosensor plate. Plates were covered and fluorescence in each well was recorded over time with a Tecan SAFIRE multimode microplate spectrophotometer. The averages are plotted, and vertical lines represent the SEM of four independent experiments performed in triplicate. The number of cells contained in equal volumes was not statistically significantly between conditions. 


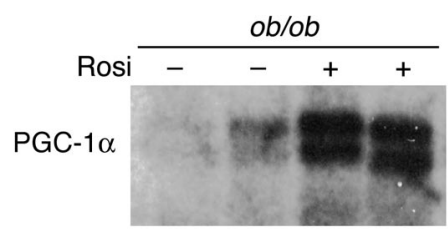

\section{Figure 10}

PGC-1 $\alpha$ expression in epididymal fat. Total RNA was prepared from fat pads from ob/ob mice before (-) and after (+) rosiglitazone treatment and was subjected to Northern blotting with a ${ }^{32}$ P-labeled mouse PGC-1 cDNA fragment as a probe.

were obtained as described previously (5). For live cell imaging, isolated adipocytes were incubated at $37^{\circ} \mathrm{C}$ for 30 minutes with $100 \mathrm{nM}$ MitoTracker Green FM (Molecular Probes), a mitochondria-specific dye, and a single in-focus optical section was acquired. The fluorescence intensity of each cell was quantified with Photoshop 7 software by analyzing randomly selected regions of equal size within a single optical section using the marquee tool. Alternatively, stacks were projected into a single image plane and the total intensity per cell was recorded. The histogram function was then used to determine the number of pixels in the selected area, and this number was multiplied by the average pixel intensity. The average of these measurements was used to compare the average intensity among different cells.

RNA preparation for Affymetrix GeneChips. Total RNA was used for the preparation of cRNA from primary fat cells isolated from 4-week-old and 26-week-old mice and 26-week-old rosiglitazone-treated mice for use with MG-U74v2 GeneChips (Affymetrix). RNA from primary fat cells was prepared from three groups of 2-4 mice for each condition. Affymetrix protocols were followed for the preparation of cRNA from the mRNA or total RNA, which was hybridized according to Affymetrix instructions. The GeneChips were washed with a GeneChip Fluidics Station 400 and were scanned with an HP GeneArrayScanner (Affymetrix). The GeneChips were analyzed with MAS 5.0 (Affymetrix) and Microsoft Excel. Array data were sorted with Affymetrix Data Mining Tool Software and Microsoft Excel spreadsheets. Results analyzed are a composite of three independent experiments. The expression level was determined using the average difference calculated by Affymetrix Microarray Suite 5.0 software. The "fold change" for each gene between 4-week-old $o b / o b$ mice and 26-week-old untreated $o b / o b$ mice was determined by dividing the mean of the average difference from three independent experiments of 4-week-old old $o b / o b$ mice by the mean of the average difference from three independent experiments of 26 -week-old untreated $o b / o b$ mice. The "fold change" for each gene between 26-week-old untreated $o b / o b$ mice and 26-week-old rosiglitazone-treated $o b / o b$ mice was determined by dividing the mean of the average difference from three independent experiments of 26-week-old $o b / o b$ mice by the mean of the average difference from three independent experiments of 26-week-old rosiglitazone-treated $o b / o b$ mice.

Northern blotting of PGC-1 $\alpha$. C57BL/6J ob/ob mice 11 weeks of age were housed ( $n=4$ per cage) under 12-hour light/dark cycles with ad libitum access to food and water. Animals were "mock-dosed" with vehicle alone by oral gavage for 4 days and then treated with either vehicle or $4 \mathrm{mg} / \mathrm{kg} /$ day of rosiglitazone in $0.25 \%$ weight/volume methylcellulose every day for 4 days. Epididymal white adipose depots were dissected and immediately homogenized in TRIzol reagent (Invitrogen Life Technologies). Total RNA was prepared with TRIzol reagent according to the manufacturer's instructions. RNA $(10 \mu \mathrm{g})$ was analyzed by Northern blotting with a ${ }^{32} \mathrm{P}$-labeled mouse PGC-1 cDNA fragment as a probe. The probe was generated by PCR of mouse adipose tissue cDNA using the following primers: PGC1F, $5^{\prime}$-CAAACCCACAGAAAACAGGAA-3', and PGC1R, 5'-TCCGTTTTGGAATTGAGTGAC- $3^{\prime}$. The PCR product was cloned into the PCR2.1-TOPO vector (Invitrogen) and the cDNA fragment was subsequently excised by digestion with EcoRI. The DNA probe was then radiolabeled with Ready-To-Go DNA Labelling Beads (-dCTP) (Amersham Biosciences) according to the manufacturer's instructions and was purified with MicroSpin G-50 columns (Amersham Biosciences). Hybridization and washes were performed with QuikHyb solution (Stratagene) according to the manufacturer's instructions.

Mitochondrial isolation. Adipocytes were rinsed twice in PBS and twice in isolation buffer ( $250 \mathrm{mM}$ sucrose, $0.5 \mathrm{mM}$ EGTA, and $5 \mathrm{mM}$ HEPES, $\mathrm{pH}$ 7.4). Cells were homogenized by ten "passes" of a motor-driven homogenizer $(500 \mathrm{rpm})$ and were centrifuged at $500 \mathrm{~g}$ for 10 minutes. The post-nuclear supernatant was removed and was centrifuged at $18,000 \mathrm{~g}$ for 25 minutes. The pellet was resuspended in $20 \%$ sucrose, $10 \mathrm{mM}$ Tris, and $0.1 \mathrm{mM}$ EDTA and was centrifuged at $18,000 \mathrm{~g}$ for 30 minutes. The pellet was resuspended in $60 \%$ sucrose, $10 \mathrm{mM}$ Tris, and $0.05 \mathrm{mM}$ EDTA. The suspension was overlaid with a 53\% sucrose layer (sucrose, $10 \mathrm{mM}$ Tris, and $0.05 \mathrm{mM}$ EDTA) and a $44 \%$ sucrose layer (sucrose, $10 \mathrm{mM}$ Tris and $0.05 \mathrm{mM}$ EDTA). The sucrose step gradient was centrifuged at $141,000 \mathrm{~g}$ for 2 hours. Purified mitochondria settled at the 44/53 interface. The mitochondrial layer was removed, diluted in isolation buffer, and centrifuged at $18,000 \mathrm{~g}$ for 30 minutes. The mitochondrial pellet was suspended in $50 \mu \mathrm{l}$ of isolation buffer and a protein determination assay was performed with the Bradford assay (Bio-Rad Laboratories). 3-[(3-cholamidopropyl)dimethylammonio]1-propanesulfonate (CHAPS) detergent was added to the suspension to a final concentration of $6 \mathrm{mM}$ CHAPS, the suspension was vortexed, and isolation buffer was then added to bring the final concentration of CHAPS to $3 \mathrm{mM}$. The suspension was vortexed continuously for 10 minutes, layered on top of a $1.9-\mathrm{ml} 10-30 \%$ sucrose gradient, and spun at $114,000 \mathrm{~g}$ in a SW 50.1 rotor (Beckman) at $4{ }^{\circ} \mathrm{C}$ for 20 hours. The gradient was fractionated into ten $200-\mu \mathrm{l}$ fractions, which were then analyzed by SDS-PAGE. Gels were then analyzed with Sypro Ruby protein stain (Molecular Probes).

Mass spectrometry. Proteins resolved by SDS-PAGE and visualized by Sypro Ruby protein stain were excised from the polyacrylamide gel and were digested in-gel with trypsin. The samples were analyzed by either electrospray ion-trap (ESI) mass spectrometry or matrix-assisted laser desorption ionization time-of-flight (MALDI-TOF) mass spectrometry. For analysis by ESI mass spectrometry, the digests were "run out" on a LC Packings UltiMate Nano HPLC (Mass Spectrometry International Ltd.) with a $100-\mu \mathrm{m}$ C18PM column in a solvent A $(0.1 \%$ formic acid and $3.5 \%$ acetonitrile) and solvent $\mathrm{B}(0.1 \%$ formic acid in a $70: 30$ mixture of acetonitrile and water). Peptides were eluted with a linear gradient from $100 \%$ solvent A to $60 \%$ solvent B in 40 minutes at a flow rate of 500 nanoliters/minutes. Peptides were eluted directly into the LCQ Deca ESI (LC/ MS) mass spectrometer equipped with data-dependent acquisition and a high resolution scan performed. A higher-energy tandem mass spectrometry (MS/MS) scan was performed after the initial scan for verification of peptide identification. The Affymetrix GeneChip database was searched for information on the presence and abundance of cDNAs encoding for the peptides identified in our study using the Sequest software developed by John Yates and Jimmy Eng at the University of Washington (Seattle, Washington, USA). For analysis by MALDI-TOF, the digested samples were further concentrated and desalted with Millipore Zip Tip C18 microtips. Peptide masses were determined with a Kratos Analytical Axima CFR MALDI-TOF spectrometer equipped with a curved field reflectron. Peptide masses were "searched against" the nonredundant protein database using the Protein Prospector program MS-Fit, a program available at the website of the Mass Spectrometry Facility of the University of California at San Francisco. Fragmentation data from individual peptides via post-source decay analysis were "searched against" the nonredundant protein database using the Protein Prospector program MS-Tag. 
Western blot analysis. Total cellular protein extracts were obtained by incubation of cells in 1\% SDS in PBS and vortexing. Protein determination assays were performed with the Bradford assay (Bio-Rad). Equal concentrations of proteins were separated by SDS-PAGE and transferred to a nitrocellulose membrane, which was blocked with $5 \%$ milk in Tris-buffered saline with Tween-20 and incubated with indicated primary antibodies (Figure 7). Membranes were then incubated with HRP-conjugated goat anti-mouse or anti-rabbit, detected by the Renaissance enhanced luminol reagent (NEN Life Science Products).

Fatty acid oxidation assay. Isolated adipocytes were washed in KRH plus $1 \%$ BSA buffer. Fresh buffer was added to the cells at a volume of two times the packed cell volume. Cells and buffer were inverted several times to make a homogenous mixture and equal aliquots of each sample were distributed into $50-\mathrm{ml}$ polystyrene tubes. $\left[{ }^{14} \mathrm{C}\right]$ palmitic acid (Perkin Elmer Life Sciences) was added at a final concentration of $200 \mathrm{nCi} / \mathrm{ml}$. An uncapped Eppendorf tube containing a piece of No. 1 Whatman filter paper soaked in $300 \mu \mathrm{l}$ benzethonium hydroxide (Sigma-Aldrich) was placed inside each $50-\mathrm{ml}$ tube. Tubes were then sealed and incubated at $37^{\circ} \mathrm{C}$ for 2 hours. Tubes were gently shaken every 30 minutes during the incubation. After 2 hours, $12 \mathrm{M} \mathrm{HCl}$ was added to the cells to release the $\left[{ }^{14} \mathrm{C}\right] \mathrm{O}_{2}$, and each tube was resealed and incubated at $37^{\circ} \mathrm{C}$ overnight. The $\left[{ }^{14} \mathrm{C}\right] \mathrm{O}_{2}$ released was measured by scintillation counting of the filter paper.

Oxygen consumption. Adipocytes isolated from 4-week-old ob/ob mice and 26-week-old untreated or rosiglitazone-treated $o b / o b$ and C57BL/6J mice were washed in KRH buffer plus 1\% BSA. Excess buffer was removed and $75 \mu \mathrm{l}$ of floating packed cells from each condition were divided into aliquots in a BD Oxygen Biosensor System plate (BD Biosciences) in triplicate. Plates were sealed and "read" on a SAFIRE multimode microplate spectrophotometer (Tecan) at 1-minute intervals for 60 minutes at an excitation wavelength of $485 \mathrm{~nm}$ and emission wavelength of $630 \mathrm{~nm}$.

Statistical analysis. Results were compared with Student's $t$ test. $P$ values less than 0.05 were considered statistically significant.

\section{Acknowledgments}

We thank Aldo Rossini for providing rosiglitazone maleate and for helpful comments during the preparation of this work. We are indebted to Dave Lambright and members of his laboratory for generously allowing us to use space, equipment, and supplies for the oxygen consumption measurements described in this article. This work is supported by NIH grant DK6083702 (to S. Corvera).

Received for publication March 30, 2004, and accepted in revised form July 27, 2004.

Address correspondence to: Silvia Corvera, Program in Molecular Medicine, 373 Plantation Street, Worcester, Massachusetts 01601, USA. Phone: (508) 856-6898; Fax: (508) 856-1617; E-mail: silvia.corvera@umassmed.edu.
1. Argyropoulos, G., and Harper, M.E. 2002. Uncoupling proteins and thermoregulation. J. Appl. Physiol. 92:2187-2198.

2. Cannon, B., and Nedergaard, J. 2004. Brown adipose tissue: function and physiological significance. Physiol. Rev. 84:277-359.

3. Rajala, M.W., and Scherer, P.E. 2003. Minireview: the adipocyte-at the crossroads of energy homeostasis, inflammation, and atherosclerosis. Endocrinology. 144:3765-3773.

4. Friedman, J.M. 2002. The function of leptin in nutrition, weight, and physiology. Nutr. Rev. 60:S1-S14; discussion S68-S84, S85-S17.

5. Wilson-Fritch, L., et al. 2003. Mitochondrial biogenesis and remodeling during adipogenesis and in response to the insulin sensitizer rosiglitazone. Mol. Cell. Biol. 23:1085-1094.

6. Olswang, Y., et al. 2002. A mutation in the peroxisome proliferator-activated receptor gamma-binding site in the gene for the cytosolic form of phosphoenolpyruvate carboxykinase reduces adipose tissue size and fat content in mice. Proc. Natl. Acad. Sci.U. S. A. 99:625-630.

7. Franckhauser, S., et al. 2002 . Increased fatty acid reesterification by PEPCK overexpression in adipose tissue leads to obesity without insulin resistance. Diabetes. 51:624-630.

8. Okuno, A., et al. 1998. Troglitazone increases the number of small adipocytes without the change of white adipose tissue mass in obese Zucker rats. J. Clin. Invest. 101:1354-1361.

9. Zhou, Z., et al. 2003. Cidea-deficient mice have lean phenotype and are resistant to obesity. Nat. Genet. 35:49-56.

10. van der Leij, F.R., et al. 2002. Structural and functional genomics of the CPT1B gene for muscletype carnitine palmitoyltransferase I in mammals. J. Biol. Chem. 277:26994-27005.

11. Brown, N.F., et al. 1997. Mouse white adipocytes and 3T3-L1 cells display an anomalous pattern of carnitine palmitoyltransferase (CPT) I isoform expression during differentiation. Inter-tissue and inter-species expression of CPT I and CPT II enzymes. Biochem. J. 327:225-231.

12. White, R.A., Dowler, L.L., Angeloni, S.V., and Koeller, D.M. 1996. Assignment of Etfdh, Etfb, and Etfa to chromosomes 3, 7, and 13: the mouse homologs of genes responsible for glutaric acidemia type II in human. Genomics. 33:131-134.

13. Stumvoll, M. 2003. Thiazolidinediones-some recent developments. Exp. Opin. Investig. Drugs. 12:1179-1187.

14. Viberti, G.C. 2003. Rosiglitazone: potential beneficial impact on cardiovascular disease. Int. J. Clin. Pract. 57:128-134.

15. Wang, T., Zang, Y., Ling, W., Corkey, B.E., and Guo, W. 2003. Metabolic partitioning of endogenous fatty acid in adipocytes. Obes. Res. 11:880-887.

16. Reshef, L., et al. 2003. Glyceroneogenesis and the triglyceride/fatty acid cycle. J. Biol. Chem. 278:30413-30416.

17. Orci, L., et al. 2004. Rapid transformation of white adipocytes into fat-oxidizing machines. Proc. Natl. Acad. Sci. U. S. A. 101:2058-2063.

18. Watkins, S.M., Reifsnyder, P.R., Pan, H.J., German, J.B., and Leiter, E.H. 2002. Lipid metabolome-wide effects of the PPARgamma agonist rosiglitazone. J. Lipid Res. 43:1809-1817.

19. Akazawa, S., Sun, F., Ito, M., Kawasaki, E., and Eguchi, K. 2000. Efficacy of troglitazone on body fat distribution in type 2 diabetes. Diabetes Care. 23:1067-1071.

20. Kelly, I.E., Han, T.S., Walsh, K., and Lean, M.E. 1999. Effects of a thiazolidinedione compound on body fat and fat distribution of patients with type 2 diabetes. Diabetes Care. 22:288-293.

21. Miyazaki, Y., et al. 2002. Effect of pioglitazone on abdominal fat distribution and insulin sensitivity in type 2 diabetic patients. J. Clin. Endocrinol. Metab. 87:2784-2791.
22. Nakamura, T., et al. 2001. Thiazolidinedione derivative improves fat distribution and multiple risk factors in subjects with visceral fat accumulation-double-blind placebo-controlled trial. Diabetes Res. Clin. Pract. 54:181-190.

23. Virtanen, K.A., et al. 2003. Differential effects of rosiglitazone and metformin on adipose tissue distribution and glucose uptake in type 2 diabetic subjects. Diabetes. 52:283-290.

24. Yoshida, Y., et al. 2002. A peroxisome proliferatoractivated receptor gamma agonist influenced daily profile of energy expenditure in genetically obese diabetic rats. Jpn. J. Pharmacol. 88:279-284.

25. Mootha, V.K., et al. 2003. PGC-1alpha-responsive genes involved in oxidative phosphorylation are coordinately downregulated in human diabetes. Nat. Genet. 34:267-273.

26. Patti, M.E., et al. 2003. Coordinated reduction of genes of oxidative metabolism in humans with insulin resistance and diabetes: Potential role of PGC1 and NRF1. Proc. Natl. Acad. Sci. U. S. A. 100:8466-8471.

27. Petersen, K.F., et al. 2003. Mitochondrial dysfunction in the elderly: possible role in insulin resistance. Science. 300:1140-1142.

28. Kelly, D.P., and Scarpulla, R.C. 2004. Transcriptional regulatory circuits controlling mitochondrial biogenesis and function. Genes Dev. 18:357-368.

29. Wu, Z., et al. 1999. Mechanisms controlling mitochondrial biogenesis and respiration through the thermogenic coactivator PGC-1. Cell. 98:115-124.

30. Puigserver, P., et al. 1998. A cold-inducible coactivator of nuclear receptors linked to adaptive thermogenesis. Cell. 92:829-839.

31. Rossmeisl, M., et al. 2002. Expression of the uncoupling protein 1 from the aP2 gene promoter stimulates mitochondrial biogenesis in unilocular adipocytes in vivo. Eur. J. Biochem. 269:19-28.

32. Tiraby, C., et al. 2003. Acquirement of brown fat cell features by human white adipocytes. J. Biol. Chem. 278:33370-33376. 